\title{
Mutations of IDH1 and IDH2 are not detected in brain metastases of colorectal cancer
}

\author{
Matthias Holdhoff · D. Williams Parsons • \\ Luis A. Diaz Jr.
}

Received: 16 February 2009/ Accepted: 16 March 2009/Published online: 7 April 2009

(C) Springer Science+Business Media, LLC. 2009

\section{To the Editor:}

Somatic mutations of codon 132 of isocitrate dehydrogenase 1 (IDH1) were recently described in a genome-wide analysis of human glioblastoma multiforme (GBM) [1]. IDH1 is involved in the production of nicotinamide adenine dinucleotide phosphate (NADPH) by catalyzing the oxidative carboxylation of isocitrate to $\alpha$-ketoglutarate. While only about $12 \%$ of the analyzed GBMs were found to have a mutation of IDH1, this alteration was detected in most secondary GBMs, and it appeared to be associated with comparatively younger age and improved survival. Mutations of IDH1 were also found in high frequency (over $70 \%$ ) in WHO grades II and III astrocytomas and oligodendrogliomas $[2,3]$. Tumors without mutations of IDH1 were often found to have mutations at the analogous position R172 of the closely related IDH2 gene [2]. These data suggest that mutations in IDH1 and IDH2 play an important role in the evolutionary, stepwise development of astrocytomas and oligodendrogliomas. Mutations of IDH, however, were not found in other solid tumors including colorectal cancer, prostate, breast and lung cancer $[2,4]$.

Hypothesizing that the selection pressure in the brain environment warrants certain constitutional adjustments of the cancer cells including mutational changes, we tried to

M. Holdhoff $(\bowtie) \cdot$ D. W. Parsons · L. A. Diaz Jr. Ludwig Center for Cancer Genetics and Therapeutics, Sidney Kimmel Comprehensive Cancer Center at Johns Hopkins, 1650 Orleans Street, Suite 590, Baltimore, MD 21231, USA

e-mail: mholdho1@jhmi.edu

D. W. Parsons

Department of Pediatrics, Baylor College of Medicine,

Houston, TX, USA address the question of whether mutations of IDH1 and IDH2 might be required for the development of brain metastasis in non-primary CNS tumors.

We analyzed tissue sections of brain metastases of seven patients with metastatic colorectal cancer. The slides were reviewed together with a neuro-pathologist and areas of metastatic colorectal cancer were extracted using the lasercapture microdissection technique. The dissected material was digested overnight with proteinase $\mathrm{K}$ (Invitrogen), and DNA was purified using the QIAmp Micro Kit (QIAGEN).

Exon 4 of the IDH1 gene and exon 4 of the IDH2 gene (containing IDH1 residue 132 and IDH2 residue 172, respectively) were PCR-amplified and sequenced as previously described [1].

None of the seven analyzed samples showed mutations in either the R132 residue of IDH1 or the R172 residue of IDH2, suggesting that these mutations are not required for the development of brain metastasis in these tumors.

\section{References}

1. Parsons DW, Jones S, Zhang X et al (2008) An integrated genomic analysis of human glioblastoma multiforme. Science 321:18071812. doi:10.1126/science. 1164382

2. Yan H, Parsons DW, Jin G et al (2009) IDH1 and IDH2 mutations in gliomas. N Engl J Med 360:765-773

3. Balss J, Meyer J, Mueller W et al (2008) Analysis of IDH1 codon 132 mutation in brain tumors. Acta Neuropathol 116:597-602

4. Bleeker FE, Lamba S, Leenstra S et al (2009) IDH1 mutations at residue p.R132 (IDH1(R132)) occur frequently in high-grade gliomas but not in other solid tumors. Hum Mutat 30:7-11. doi: 10.1002/humu.20937 